\title{
Light in the Service of Mankind
}

\author{
Jean-Patrick Connerade
}

Quantum Optics and Laser Science Group, Physics Department, Imperial College, London and European Academy of Sciences Arts and Literature, UK

Copyright $\subseteq 2017$ by authors, all rights reserved. Authors agree that this article remains permanently open access under the terms of the Creative Commons Attribution License 4.0 International License

\begin{abstract}
Understanding the nature of light has stimulated scientific research over many centuries and progress in the description of electromagnetic radiation has been key in the development of modern science. Some highlights in this story are celebrated in the context of the International Year of Light YL2015 declared by UNESCO, which were the backdrop for scientific meetings held in Caceres (Spain) and in Poland (Wroclaw) involving the European Academy of Sciences Arts and Letters. It is emphasised that this long history of research on Light is by no means over, and has resulted in many important advances, including such applications as the laser and the harnessing of solar energy for electric power generation, of great importance for the economic development of Southern regions such as North Africa. Cultural aspects of the importance of light (in the Arts, in literature and in philosophy) are also mentioned.
\end{abstract}

Keywords Light, Radiation, Laser, Solar Energy, Development, Africa, European Academy, UNESCO

\section{Introduction}

The present paper was presented at a meeting in Wroclaw [1] in the year 2015 - chosen by UNESCO as the year for the worldwide celebration of Light. 'Celebration' of course implies that we should re-visit all the various benefits which have come from light and attempt to remember them and discuss their importance. As physicists, chemists and spectroscopists, we have of course much to contribute to this fundamental question, but it is not the prerogative of scientists to take the whole subject over. Philosophers, historians, painters, sculptors and indeed artists in general, not to forget the engineers involved in harnessing solar radiation for our benefit, would also claim that light is the key to their activities. Keeping the theme in the singular, theologians would also point to the amazingly perceptive phrase «Fiat lux...» (Let there be light) as marking the origin of the universe. And if we put the subject in the plural, then we would probably agree collectively that the modern democracies of the Western world are all somehow issued from Le siècle des lumières, which is how, in French, one describes the age of Voltaire and the philosophers of the eighteenth century, whose influence was so great that it was subsequently likened to beacons of light illuminating modern times. This translates into English as the Age of Enlightenment.

Thus, the theme of light, taken in its broadest context which is very much in the spirit of UNESCO, is all pervasive. The European Academy of Sciences Arts and Literature (EASAL) comes under the cultural aegis of UNESCO, and so it seemed most appropriate that we should prepare the advent of the year of light by organising a conference under the general title Light in the service of mankind, staged in a city called Cáceres, itself belonging to the World Cultural Heritage owing to the beauty of its monuments and to its past stretching back through Spanish and Arabian back to Roman times.

The purpose of this earlier meeting in Spain meeting was to cover as many as we could of the themes just mentioned, extending from physics and astronomy through history, philosophy, the arts and solar power generation, which is of course a subject of tremendous importance in the whole of the Mediterranean basin (I will come back to that). Many members of EASAL attended the international colloquium in Cáceres. I should mention in particular the presence a very distinguished member: the Nobel Laureate Professor Claude Cohen-Tannoudji of the Collège de France, who is of course one of the world's greatest experts on the subject of Light, as regards its fundamental properties and its use in atomic cooling experiments.

The meeting in Cáceres took place just before another conference, held in Lisbon, organised by a Foundation based around CERN in Geneva [2], whose activities are very much along the same lines as those of EASAL [3] in the Mediterranean area, namely the concept of reaching across the zone by sharing the benefits of scientific and technical innovation. Many of the themes discussed in Cáceres also featured prominently in the Lisbon Conference. The reason for mentioning it here is again that it belongs to a growing number of conferences whose avowed purpose is to re-create something like the spirit of the Age of Enlightenment and 
open up the boundaries of knowledge between the peoples of the world rather than treat knowledge as a source of ever increasing revenue for the few who possess it all.

\section{How Light and Knowledge Became Intertwined}

I return now to the subject of light, and would like to stress how the attempt to understand the enigmatic nature of light has accompanied human knowledge since the very beginnings of civilisation. If we consider what Feynman himself regarded as the most important statement in Science (the one, as he puts it [4], which should be preserved if some disaster were to strike the whole of humanity), namely the atomic hypothesis, first formulated by Democritus more than two thousand years ago, it is very interesting that, as soon as the existence of the atom as the fundamental building block of matter was postulated, the question arose: what should there be between the atoms. Democritus himself provided the most elegant answer: between the atoms, there is nothing. Thus, together with the atoms, the vacuum was born, and Democritus should rightfully be regarded as its discoverer. Originally, the vacuum was very simple: it consisted merely in the complete absence of atoms (i.e. of all matter). However, as science progressed, an extraordinary mystery emerged. It became clear that, above the earth's atmosphere [5], there exists such a vacuum (and a black sky [6]), but that, somehow, the light from the sun and indeed from distant stars can travel through this vacuum to reach us [7]. So, the situation was not quite as simple as Democritus had supposed. No doubt, the vacuum is empty of matter but, in spite of this emptiness, can transport light. This was so extraordinary that it became necessary to postulate the existence of the ether [8] - an all-pervasive medium whose prime function would be allowing light waves to reach us. All physicists know that the properties of the ether, an apparently undetectable fluid supporting the propagation of electromagnetic waves, kept Maxwell [9] and others awake at night pondering on its mysteries, and that the climax came with was the Michelson-Morley experiment [10], leading to the formulation of the Lorentz transformations [11], the theory of Relativity [12] and, perhaps more strangely, to the tacit assumption that the ether is completely unobservable or, as some would say, simply does not exist.

One might at first think that it would be difficult to find anything else quite as fundamental as these advances. Yet, others were in course. When Newton considered the properties of light, he could not himself decide whether it should be treated as a wave or as what he called a 'corpuscle' [13], namely a particle, and this ambiguity persisted throughout his scientific work. Sometimes, he used one description but, for other problems, he used the other, without seemingly worrying about any contradiction between the two. Throughout the eighteenth and nineteenth centuries, many experiments were done to resolve this ambiguity, resulting in the huge development of physical optics in the hands of Fresnel [14], Young [15], Huygens [16] and many others and in the discovery of electromagnetism by Faraday [17] and Maxwell [18], which reinforced the opinion that light was in fact nothing else but an electromagnetic wave. There matters stood until Louis de Broglie [19] saw the correspondence between optics and mechanics and realised that the sharp distinction between wave and particle was itself incorrect. It is quite reasonable to consider that most of quantum mechanics emerged from studies of atoms using light (spectroscopy) and from de Broglie's wave-particle duality, which suddenly restored Newton's view that light should be considered as both wave and 'corpuscle'. It was left to Einstein to describe the fundamental properties of what today we call a photon [20], but which in principle is simply Newton's 'corpuscle' reborn.

It is often argued that Young's double-slit experiment 'proves' the wave nature of light. This of course is true, but it does not exclude the 'particle' nature of light (i.e. the photon). It is merely that proving the existence of the photon experimentally is much more difficult. Indeed, early attempts were made to reconcile the wave nature of light with quantum mechanics by assuming that light was purely a wave and that only the absorption and emission needed to be quantised. This, however, is not the case, but is hard to establish experimentally. The consensus amongst physicists is that the definite experimental proof of the existence of the photon was finally achieved in 1977 in the photon anti-bunching experiment in resonance fluorescence by Kimble Dagenais and Mandel [36]. Today, we see that the distinction between particles and waves, which de Broglie had banished from physics, does not simplify the situation. It is replaced by another, the distinction between the fundamental fermions and bosons, in which photons possess integral spin like all the particles which 'mediate' or transport fields, whereas half-integral spin characterizes the particles which 'experience' the fields [20]. The vacuum is no longer 'empty' but contains a sea of 'virtual' particles which are supposed to assist the propagation of waves, including light. Such descriptions can be given beautiful mathematical forms, but in essence, they do remain mysterious as to how they operate. Although they seem familiar to manipulate, they remain as strange in principle as the 'aether' of ancient times.

But this is still not the end of the story. Many other surprises have come from the study of light. Already, Laplace, in his famous Mécanique Céleste [21] had wondered whether gravity might somehow influence the propagation of light. At first sight, this might seem impossible in classical physics, since light has no mass. However, Laplace had noticed that, in the formula for the escape velocity (which we describe today as the minimum velocity for a rocket to escape from the attraction of a planet [22]), the mass of the escaping particle actually cancels out of the equation. So, he had the brilliant idea of introducing 
Newton's corpuscle of light as the escaping particle and, since the velocity of light is fixed, asking the opposite question: how dense should a star be for no light to be able to escape from it? From this very simple (and completely classical) argument, he obtained a formula for what we call today the Schwarzschild radius [23]. In fact, he had just invented the black hole [24].

At the time, nobody took his argument seriously. Fresnel [14] (it was thought) had proved conclusively that light was a wave. So, while Laplace's magnificent book was universally admired, these few pages came to be regarded as the aberrations of a great mind who could not accept that Newton might have been wrong about corpuscles, which is why the official discovery of black holes had to await the advent of relativity [24].

When Hubble [25] uncovered (this time by using light as a wave emitted by distant galaxies and considering Doppler shifts) the expansion of the Universe and established the reality of the 'Big Bang' theory [26], it became clear that the concept of a black hole is somehow related to the earliest moments of the world as we know it. It became an all-important object of study for many theoretical physicists. The general public soon heard about it and even began to worry about whether some mad scientist might be able to make one and release it from his laboratory. Fortunately, there is not much danger of that, although it soon emerged that black holes really do exist [27] (luckily, very far away from us) and that their properties are truly terrifying.

Indeed, the connection between radiation and the first instants of the 'Big Bang' became much clearer with the first observation of what is called fossil radiation namely the residual light of the Big Bang or the 4 degree background, observed experimentally. Studying the properties of this light, and in particular its distribution over the celestial sphere, yields fundamental information on the universe as it was in its first observable moments, just after the 'Planck time' i.e. $10^{-44}$ seconds after the Big Bang [28]. What is now regarded as the 'first observable instant' of the universe-described-by-the-laws-of-physics is the instant when light separated from matter, and further evidence for this process had been claimed quite recently from studies of astronomical data (the BICEP2 dataset) although the conclusions have since been withdrawn as an artifact due to interstellar dust [29]. The quest for such evidence through astronomy will obviously continue in view of the huge interest any observation of this kind generates.

What also clear is that the properties of light will continue to play a fundamental role in understanding the physics of the early universe. To take but one example, the Planck time, if one interprets it as the shortest possible interval, sets a fundamental limit also to the frequency of any kind of wave, viz. the inverse of the Planck time, which thus appears as the very highest frequency which can possibly be observed.

It seems, therefore, whenever one asks truly fundamental questions in physics, that they can only be answered by understanding better the properties of light.

\section{Applications of Light}

This, however, still does not complete the story. Once the properties of light are better understood, it becomes possible to make use of them in new ways. Thus, Einstein introduced the photon [30], and this idea led him to the concept [31] of the spontaneous emission of light by atoms, a new process which competes with the more classical emission of light by excited atoms when stimulated by a perturbation. The study of this competition between spontaneous and stimulated emission led Kastler [32] to the discovery of a process called optical pumping and hence to the invention of the laser [33], one of the most important tools in science and in modern engineering. The laser is indeed the foremost example of a device invented as the application of a piece of fundamental research. It started life as a toy for scientists to play with, but now the world is full of lasers being used for one purpose or another, to read or transport information, to generate ultrashort pulses, to melt materials, to level agricultural land to digitize the shapes of buildings, to clean pollution off stones, to remove old varnish from paintings, etc. etc. Lasers have brought us coherent light, a very special and most useful kind of radiation, different in most of its properties from the 'ordinary' light we were familiar with before.

I promised also some comments regarding applications of light in the service of man in the Mediterranean region where the Cáceres conference was held. In much of North Africa, and indeed also in parts of Southern Europe, there is a serious shortage of fresh water. Water is essential for agriculture, for tourism and to develop new cities. In fertile areas of Morocco, for example, so much water has been pumped up for irrigation in order to grow fresh vegetables in the early spring (one of the mains exports of the country) that the water table has sunk down to some forty metres below ground level. When falls to such a low level, the salt content rises and the water becomes brackish. Not only is this a problem for access to drinking water of the local inhabitants, but it also impedes agriculture and tourism, which are the principal economic activities, and prevents the creation of new coastal cities, in spite of an ever-increasing demographic pressure [34] The economic development of North Africa depends critically on desalination as the major new source of fresh water. In principle, the techniques of osmosis developed in recent years have brought prices down to a competitive level, but the problem now is to find energy sources to power the large plants which will be necessary. The best local source of energy is of course sunlight, which is abundant in the region and non-polluting. Indeed, research into the generation of energy from solar radiation presents a unique opportunity for Southern countries to gain a leading position by using their climatic advantage. Spain is already in an enviable position in Europe, and many countries of North Africa have fast-developing programmes [35]. The next step would clearly be to harness this form of energy to a desalination plant. Basically, this is just copying nature. Quite clearly, this is where the effort must go, and this is also where sharing 
knowledge across the Mediterranean might make a difference, not only in terms of advancing joint research, but also in building trust between nations of the region via scientific and technical collaboration. Hopefully, in the future, light will also be seen as a means of overcoming poverty by enabling modern development.

\section{Light in the Arts and 'Enlightenment'}

I have not, so far, said anything about the arts, so let me comment briefly on this crucial aspect.

Light, of course, is essential to painters. The conference in Cáceres was held in the year of the four hundredth anniversary of the death of the great painter El Greco, a true representative of the Mediterranean spirit, since he was originally a Byzantine Greek, travelled to Renaissance Italy and settled finally in the Andalusian city of Toledo. There is a nice story about Clovio visiting El Greco on a summer's day while the artist was still living in Rome. He found El Greco sitting in a darkened room, because he experienced darkness as more conducive to thought than the light of day, which disturbed his own "inner light". This use of the word light to represent higher aspirations of man is of course what is also implied by referring to the philosophy of Les Lumières (the age of Enlightenment), I mentioned at the start of this talk. This movement is of great importance in Western culture: it led to the separation between the Church and the State, and eventually to the conceptual framework of contemporary Western democracies.

It was pointed out by the philosopher Sadik Al Azm from Damascus (a recipient of the Erasmus prize for philosophy) that a comparable movement has never yet occurred in the cultures of many south Mediterranean countries [36]. It is hardly an exaggeration to say that this troublesome fact causes huge political difficulties in the world today. In plain words, the root of our problems, in many ways, is the need to bring the spirit of the age of Enlightenment across cultural borders between the North and South Mediterranean. If this could only be achieved, it would bring prosperity and progress to a part of the world which is fundamentally very rich in its cultural heritage, but lacks this essential ingredient to participate fully in the modern development of our planet.

\section{REFERENCES}

[1] 2015 the XIIIth International Conference on Molecular spectroscopy Wroclaw Poland

[2] Fondation Partager le Savoir 1, Place des Florentins 1204 Geneva Switzerland, see also www.partager-le-savoir.org for more information.

[3] EASAL The European Academy of Sciences Arts and Letters (under the aegis of UNESCO) 60 rue Monsieur-le-Prince 75006 Paris, France see also the website: www.europeanacademysciencesartsandletters.com

[4] The Feynman lectures on physics Richard P Feynman, Robert B Leighton \& Matthew L Sands Reading, MA. : Addison-Wesley Pub. Co., 1963 Addison-Wesley world student series.

[5] Expériences nouvelles touchant le vide, Blaise Pascal 1647 see De Torricelli à Pascal Published in Philosophia Scientiæ 14-2 (2010)

[6] The idea of a black sky above the earth's atmosphere is already implicit in an experiment described The Notebooks of Leonardo Da Vinci (Jean Paul Richter (Translator) CreateSpace Independent Publishing Platform 2014) ISBN-13: 978-1502373151) Leonardo noted that, to reproduce the blue colour of the sky in his scattering experiment, he needed to place a piece of black velvet behind the water spray acting as the scatterer.

[7] In 1676, the Danish astronomer Ole Rømer (1644-1710) observed the orbits of Jupiter's satellites. Rømer knew that such eclipses should occur periodically, so the $\mathrm{n}^{\text {th }}$ eclipse is expected at a time $\mathrm{nP}$, where $\mathrm{P}$ is the period, but in fact this time varied according to the relative position of the Earth and Jupiter by as much as 16 minutes. This could only be due to the variation in their distance from Earth as they orbited the Sun. Rømer realized that, if light travelled at finite speed, the time to travel the extra distance would explain the variation.

[8] Einstein, Albert: Ether and the Theory of Relativity (1920), republished in Sidelights on Relativity (Methuen, London, 1922)

[9] A Treatise on Electricity and Magnetism (2-vol.) James Clerk Maxwell 1873 Reprinted Dover Books USA

[10] Michelson, Albert A. and Morley, Edward W. (1887). On the Relative Motion of the Earth and the Luminiferous Ether. American Journal of Science. 34: 333-345

[11] Lorentz, Hendrik Antoon (1895) Versuch einer Theorie der electrischen und optischen Erscheinungen in bewegten Körpern, Leiden: E.J. Brill

[12] Einstein A. (1916) Relativity: The Special and General Theory (Translation 1920), New York: H. Holt and Company

[13] Newton, Isaac (1704) Opticks: Or, a Treatise of the Reflexions, Refractions, Inflexions and Colours of Light London Sam Smith and Benjamin Walford Princes Arms St Paul's Churchyard

[14] Fresnel, Augustin Jean (1819). Memoir on the Diffraction of Light" American Book Company. pp.79-145

[15] Young, T. (1807). A Course of Lectures on Natural Philosophy and the Mechanical Arts. Vol.1. William Savage. Lecture 39, pp.463-464.

[16] Huygens Christian, Traité de la Lumière (drafted 1678; published in Leyden by Van der Aa, 1690), translated by Silvanus P. Thompson as Treatise on Light (London: Macmillan, 1912; Project Gutenberg edition, 2005), p.19.

[17] Faraday, Michael (1839) Experimental Researches in Electricity, vols. I and II Richard and John Edward Taylor.; vol. III. Richard Taylor and William Francis, 1855

[18] Maxwell, James Clerk see ref [9] 
[19] de Broglie, Louis Victor Pierre Raymond (1924) Recherches sur la théorie des quanta (Thèse) Université de Paris and Annales de Physique (10)3, 22(1925)

[20] se e.g. Ryder Lewis H (1985) Quantum Field Theory Cambridge University Press Cambridge

[21] de Laplace, Pierre Simon (1796) reprinted 1805 Traité de mécanique céleste, chez Courcier quai des Augustins No.71 Paris.

[22] see e.g. Kibble T.W.B. and Frank H. Berkshire (2004) Classical Mechanics Singapore, Imperial College Press, page 193

[23] Schwarzschild, K. (1916) Über das Gravitationsfeld eines Massenpunktes nach der Einsteinschen Theorie, Sitzungsberichte der Deutschen Akademie der Wissenschaften zu Berlin, Klasse fur Mathematik, Physik, und Technik p.189.

[24] Overbye, Dennis (2008). John Archibald Wheeler, Physicist Who Coined the Term 'Black Hole' The New York Times. "John A. Wheeler, a visionary physicist and teacher who helped invent the theory of nuclear fission, gave black holes their name and argued about the nature of reality with Albert Einstein and Niels Bohr, died Sunday morning at his home in Hightstown, N.J. He was 96."

[25] Hubble, Edwin Powell (1929) A relation between distance and radial velocity among extra-galactic nebulae. PNAS. 15 (3): $168-173$

[26] Gamow, George (1947) One Two Three... Infinity: Facts and Speculations of Science Dover Books, USA

[27] Bolton, C. T. (1972) Identification of Cygnus X-1 with HDE 226868 Nature. 235 (5336): 271-273.

[28] The Planck time is obtained from the unique combination of the gravitational constant $\mathrm{G}$, the special-relativistic constant $\mathrm{c}$, and the quantum constant $\hbar: 5.39 ? 10-44 \mathrm{~s}$. It is regarded as the shortest time accessible within the laws of Physics.

[29] Cowen, Ron (2015) Gravitational Waves Discovery Now Officially Dead Kovac and his colleagues pored over the BICEP2 dataset By Ron Cowen, Nature magazine on February 2, 2015

[30] Einstein, A. (1905). Über einen die Erzeugung und Verwandlung des Lichtes betreffenden heuristischen Gesichtspunkt Annalen der Physik 17 (6): 132-148

[31] See e.g. Milne, E. A. (1928) The effect of collisions on monochromatic radiative equilibrium, Monthly Notices of the Royal Astronomical Society, 88: 493-502. [1].

[32] Kastler Alfred (1967) Optical Methods for Studying Hertzian Resonances. Science. 158 (3798): 214-221

[33] Maiman, T.H. (1960) TC1 Stimulated Optical Emission in Ruby. Journal of the Optical Society of America. 50 (11): 1134.

[34] Farzaneh Roudi-Fahimi, Liz Creel, and Roger-Mark De Souza (2002) Finding the Balance: Population and Water Scarcity in the Middle East and North Africa Population Reference Bureau Report see: http://www.prb.org/Publications/Reports/2002/FindingtheBal ancePopulationandWaterScarcityintheMiddleEastandNorthAf rica.aspx

[35] Neslen, Arthur (2016) Morocco to switch on first phase of world's largest solar plant The Guardian Thursday 4 February 201611.47 GMT

[36] Al Azm, Sadik (2014) Critique of Religious Thought (English translation of naqd al-fikr ad-dini) Gerlach Press, Berlin and London ISBN-13: 978-3940924445

[37] Kimble HJ Dagenais M and Mandel L (1977) Phys. Rev. Lett. 39,691 